\title{
Nurses Knowledge Regarding Prevention Protocol of COVID-19 in Emergency Departments
}

\author{
Balqees Alzyoud ${ }^{1}$, Mohammed ALBashtawy², Jamal Qaddumi ${ }^{3}$, Omar Al Omar ${ }^{4}$, Shereen Hamadneh ${ }^{5}$, \\ Ayman Bani Salameh ${ }^{6}$, Abdullah Alkhawaldeh ${ }^{7}$ \\ ${ }^{1} R N$, Master Student, Critical Care Nursing, ${ }^{2}$ Professor, Princess, Salma Faculty of Nursing, AL al-Bayt University, \\ Mafraq, Jordan, ${ }^{3}$ Associate Professor, Faculty of Medicine and Health Sciences, An-Najah National University, \\ Nablus-Palestine, ${ }^{4}$ Associate Professor, Faculty of Nursing, Sultan Qaboos University, Muscat, Oman, ${ }^{5}$ Associate \\ Professor, Princess Salma Faculty of Nursing, AL al-Bayt University, Mafraq, Jordan, ${ }^{6}$ Assistance Professor, \\ Faculty of Nursing, Alzaytoonah University of Jordan, Amman, Jordan, ${ }^{7}$ Assistance Professor, Princess Salma \\ Faculty of Nursing, AL al-Bayt University, Mafraq, Jordan
}

\begin{abstract}
Corona virus disease (COVID-19) pandemic, caused by severe acute respiratory syndrome corona virus 2 (SARS-CoV-2), and is considered a global challenge.

This short review intends to investigate the knowledge of nurses regarding prevention protocol of COVID-19 in emergency departments. The searching was carried out in electronic data bases: Google scholar, PubMed and World Health Organization (WHO). Most of the reviewed studies stated a good level of knowledge, attitude and a practice among nurses regarding COVID-19. The community education is needed to improve the knowledge, practice and attitude of the people. Also, continuous training for all healthcare workers to help them to be more effective in controlling of the COVID-19 pandemic.
\end{abstract}

Keywords: Coronavirus, COVID-19, Prevention Protocol, Emergency Department.

\section{Introduction}

Novel 2019 coronavirus disease (COVID-19) is a respiratory infection breed by the virus SARS-Cov-2, which was recently detected after an outbreak began in Wuhan, China, in December $2019^{(1)}$.

The COVID-19 was stated a pandemic by the World Health Organization (WHO) on March 11, 2020, after the identification of more than 118000 cases in 114 countries ${ }^{(2)}$. The common clinical symptoms of patients with COVID-19 may include: fever, fatigue and dry cough, occasionally accompanied by nasal congestion,

\section{Corresponding Author:}

Abdullah Alkhawaldeh, $\mathrm{PhD}$,

Assistance Professor, Princess Salma Faculty of

Nursing, AL al-Bayt University, Mafraq, Jordan.

Email: dr-abd@aabu.edu.jo

abdo_752012@yahoo.com runny nose, malaise, tachypnea, shortness of breath and sore throat ${ }^{(3-5)}$. The COVID-19 is mainly transmitted through direct person-to-person transmission of severe acute respiratory syndrome coronavirus 2 (SARS$\mathrm{CoV}-2$ ), and it occurs through close-distance contact by respiratory droplets; virus released in the respiratory secretions when a person with infection coughs, sneezes, or talks can infect another person if it makes direct contact with the mucous membranes. Also, infection can occur if a person touches an infected surface and then touches his eyes, nose or mouth ${ }^{(4,6,7)}$.

The COVID-19 is spreading in our community. Person to stay safe should take some precautions such as: physical distancing (maintain at least a 1-metre distance between yourself and others to reduce your risk of infection when they cough, sneeze or speak). Wearing a mask (clean your hands before you put your mask on, as well as before and after you take it off, make sure it covers both your nose, mouth and chin), and mask 
should be changed every 6 hours or earlier if it becomes soiled, and N95 respirators may be used for suspected or confirmed positive patient ${ }^{(2)}$. Also, keeping rooms well ventilated, avoiding crowds, washing your hands for at least 20 seconds with soap and water or hand sanitizer with at least $60 \%$ alcohol, and coughing into a bent elbow, terminal cleaning was done after the patient had vacated the room, it was thoroughly cleaned before next admission. Proper biomedical waste segregation was done as per the guidelines ${ }^{(8,9)}$.

The COVID-19 is highly infectious disease, and there has not yet been any vaccine or effective treatment that has received approval. So, the best solution to avoid being exposed to the virus will be the simultaneous application of preventive methods $(10)$.

The China CDC issued a guideline to promote awareness of the prevention and control of COVID-19 among the general population. The key messages of the guideline include how to select and wear face masks, adequate hand washing habits, preventive protocol in different locations (include at home, on public transportation, and in public space), disinfection methods and medical observation at home ${ }^{(4)}$.

This short review intends to assessment the major literature thatstudy the knowledge of nurses about prevention protocol of COVID-19 in emergency department.

\section{Method}

Search methods: The electronic searching was conducted in a different database: Google scholar, Pubmed, and WHO. Key search terms used: Coronavirus COVID-19, prevention Protocol.

The studies that focused on the topic of prevention protocol of COVID-19, were published in English between 2019 and 2020 were included in the review. While, studies published before 2019 were excluded.

Search outcome: Exploring of literature yield about 50 studies for review, after reading the studies about 25 studywere excluded and 25 studies met the inclusion criteria.

\section{Discussion}

Regarding the clinical features of patients infected with 2019 novel coronavirus in Wuhan, China. Most affected patients were men had underlying diseases including diabetes, hypertension and cardiovascular disease. The common symptoms at the onset of illness were fever, cough, and myalgia or fatigue. Less common symptoms were sputum production, headache, hemoptysis and diarrhea ${ }^{(3,4)}$.

The precautions in the preventing and controlling the outbreak of COVID-19 in non-isolated areas in a general hospital were reported and it was recommended that there is no hospital-acquired COVID-19 infection among hospital staff. Also, the rates for wearing masks, checking epidemiological history and disinfecting medical supplies were $100 \%$ in the hospital. The accuracy of wearing masks among patients and their families was $73.79 \%$ and the percentage of commitment to hygiene of their hands was $40.78 \%{ }^{(4)}$.

The prevention of infection with airborne pathogens and exposure to particulates matter and aerosols (environmental pollutants and allergens) can be facilitated through the use of disposable face masks. The effectiveness of such masks at excluding pathogens and pollutants depends on the intrinsic ability of masks to resist penetration of airborne pollutants $(2,8,9)$.

The level of knowledge regarding the prevention protocol of COVID-19 was ranged between fair to good as reported in this study ${ }^{(11)}$. Healthcare worker especially nurses shave good knowledge, good practice and a positive attitude concerning COVID-19 in emergency departments. And, they perceived that poor knowledge about transmission and limited infection control material were the main barriers to infection control ${ }^{(12)}$.

The professional training that the nurses received, the level of nurse experience and education were found as factors that affecting the nurse's knowledge and practice level regarding the prevention protocol of COVID-19 ${ }^{(13,14) \text {. }}$

A recent study found gaps in specific aspects of knowledge and practice that should be focused on in any future awareness and educational campaigns. Also, the study showed that nurses were using less reliable sources of information; and this need to be addressed immediately as it ultimately affects. The health care system has to provide a comprehensive training program 
to promote all prevention protocol of COVID-19 ${ }^{(12,15)}$.

\section{Conclusion}

Most of the reviewed studies stated a good level of knowledge, attitude and a practice among nurses regarding COVID-19. The community education should have effect through a commitment to use all strategies that may improve the knowledge, practice, and of the people. Continuous training has to be provided for all healthcare workers to help them to be more effective in diagnosis and management to control such pandemic.

Conflict of Interest: No conflict of interest.

Ethical Clearance: Taken from Princess Salma Faculty of Nursing, AL al-Bayt University ethical committee.

Source of Funding: Self.

\section{References}

1. Desai AN, Patel P. Stopping the spread of COVID-19. JAMA 2020; 323:1516-1516.

2. World Health Organization. WHO declares COVID-19 a pandemic. 2020.

3. Huang C, Wang Y, Li X et al. Clinical features of patients infected with 2019 novel coronavirus in Wuhan, China. The lancet 2020; 395:497-506.

4. Mohamed K, RodríguezRomán E, Rahmani F et al. National Health commission and national administration of traditional Chinese medicine of the People's Republic of China. Protocols for diagnosis and treatment of COVID-19(5th trial version).(2020-02-05)[2020-02-06].

5. Hui DS, Azhar EI, Madani TAet al. The continuing 2019-nCoV epidemic threat of novel coronaviruses to global health-The latest 2019 novel coronavirus outbreak in Wuhan, China. International Journal of Infectious Diseases 2020; 91:264-266.

6. Chan JFW, Yuan S, Kok K H et al. A familial cluster of pneumonia associated with the 2019 novel coronavirus indicating person-to-person transmission: a study of a family cluster. The Lancet 2020; 395:514-523.
7. Li Q, Guan X, Wu P et al. Early transmission dynamics in wuhan, China, of novel coronavirusinfected pneumonia.N EnglMed 2002; 10.1056/ NEJMoa2001316.

8. Brosseau L, Ann RB. N95 respirators and surgical masks. Centers for Disease Control and Prevention. 2009 Oct.

9. De Man P, van Straten B, van den Dobbelsteen Jet al. Sterilization of disposable face masks by means of standardized dry and steam sterilization processes; an alternative in the fight against mask shortages due to COVID-19. Journal of Hospital Infection 2020; 105:356-357.

10. Momtazmanesh S, Ochs HD, Uddin LQ et al. All together to Fight COVID-19. The American Journal of Tropical Medicine and Hygiene 2020; 102:1181-1183.

11. Zenbaba D, Sahiledengle B, Bogale D. Practices of Healthcare Workers regarding Infection Prevention in Bale Zone Hospitals, Southeast Ethiopia. Advances in Public Health. 2020.

12. Saqlain M, Munir MM, Rehman SU et al. Knowledge, attitude, practice and perceived barriers among healthcare workers regarding COVID-19: a cross-sectional survey from Pakistan. Journal of Hospital Infection 2020; 105:419-423.

13. Zhou M, Tang F, Wang Yet al. Knowledge, attitude and practice regarding COVID-19 among health care workers in Henan, China. Journal of Hospital Infection. 2020

14. Zhong BL, Luo W, Li HM et al. Knowledge, attitudes, and practices towards COVID-19 among Chinese residents during the rapid rise period of the COVID-19 outbreak: a quick online cross-sectional survey. International journal of biological sciences 2020; 16: 1745 .

15. AL-rawashdeh N, Al Bashtawy M, Ozaybi N et al. Nurses Roles in Providing Care for Patient with COVID-19. EC Emergency Medicine and Critical Care 2020; 5. 